\title{
High-Efficiency Coupling-Insensitive Transcutaneous Power and Data Transmission Via an Inductive Link
}

\author{
CLEMENS M. ZIERHOFER AND ERWIN S. HOCHMAIR
}

\begin{abstract}
This paper presents a new approach for transmitting RF power and signal via an inductive link. Such an approach optimizes the power efficiency of the overall transmission scheme comprising the power amplifier plus the inductive link. Power amplification is based on the single ended class $E$ concept. The power amplification stage is self oscillating, the oscillation frequency thus being influenced by the coupling of the coils. The resulting operating frequency off set yields an improved power transmission performance of the circuit since the oscillation frequency tracks the absolute transmission efficiency maximum. A detailed analysis is given. Realization of the described approach requires a minimal number of circuit components. Experimental and theoretical results are in good agreement.
\end{abstract}

\section{INTRODUCTION}

$\mathrm{O}$ NE POSSIBILITY for providing an implanted stimulator transcutaneously with power and/or information is to transmit RF power via an inductive link. An inductive link generally consists of two resonant circuits, one external and the other as part of an implanted stimulator. The inductivities of the two resonant circuits are realized by two pancake shaped coils. When facing each other, they form a transformer which allows energy transfer from the transmitter to the implant. Inductive links have been investigated with regard to optimization of efficiency of the power transfer and the tolerance to coupling coil misalignment [1]-[5]. All these links are designed to operate at a fixed operating frequency.

The transfer resonant circuit is either series- or parallel tuned. A series-tuned transmitter requires a voltage source, whereas a parallel tuned transmitter is driven by a current source. A voltage source can be realized with two transistor switches which simply switch the input of the series tuned transmitter between the rails of the power supply (i.e., a class D amplifier, e.g., used in [2] and [6]). A single transistor can act as a current source, capable of driving a parallel tuned transmitter circuit (e.g., class $\mathrm{C}$ amplifier). In both cases radio frequency has to be generated by an RF oscillator.

Overall efficiency of a power transmission scheme is defined as the ratio of power delivered to the load (implant) and the overall dc power consumed by the whole system. The overall efficiency of a transmission scheme consisting of RF oscillator, power amplifier, and induc-

Manuscript received February 17, 1989; revised October 23, 1989

The authors are with the Inst. of Experimentalphysik, Angewandle Physik, Universitaet Innsbruck, A-6020 Innsbruck, Austria.

IEEE Log Number 9035444. tive link including load is determined by the power consumption of the RF oscillator, the efficiency of the power amplifier, and the efficiency of the inductive link itself. Earlier designs were aimed at optimizing exclusively the performance of the inductive link. This paper presents an approach for optimizing the whole transmission scheme in terms of the two criteria, overall efficiency and tolerance to coil misalignment.

Overall efficiency and tolerance to coil distance variations exert a mutual influence on one another. The electronic circuits of most implants require a sufficiently stable voltage supply to ensure optimum operation. If the transmitted RF energy is highly dependent on the mutual coil position, efficiency is noticeably reduced by power losses due to voltage regulators within the implant, which are necessary for the provision of an adequate supply voltage.

The approach used here for the realization of a coupling-insensitive high efficiency power/data link is based on the high efficiency class $\mathrm{E}$ power amplification concept. The idea was to combine a class $E$ amplifier with a tuned inductive link. In addition, the class $\mathrm{E}$ amplifier is self oscillating. Oscillation frequency is not fixed, but influenced by the mutual position of the coils. This self oscillating final stage has two basic advantages over a driven RF amplifier. First, no oscillator is necessary for generating an RF voltage, thus avoiding additional power losses. Second, oscillation frequency offset due to coupling variations significantly improves power transmission performance since the resulting oscillation frequency tracks the absolute transmission efficiency maximum, whose spectral location is dependent on the coil coupling [see Section III-A.2].

\section{Class E Tuned Power Oscillator Involving an INDUCTIVE LINK}

The fundamentals of the class $E$ power amplification concept have been broadly discussed in the literature [7][10] and application examples have been presented [11], [12]. An example of the combination of a class $E$ tuned power oscillator and an inductive link is depicted in Fig. 1.

In this case the series tuned output circuit of an ordinary class $E$ power amplifier is replaced by a stagger tuned inductive link. The inductive link is represented by a parallel resonant receiver circuit $\left(L_{2}, C_{2}, R_{2}\right)$ which is cou- 


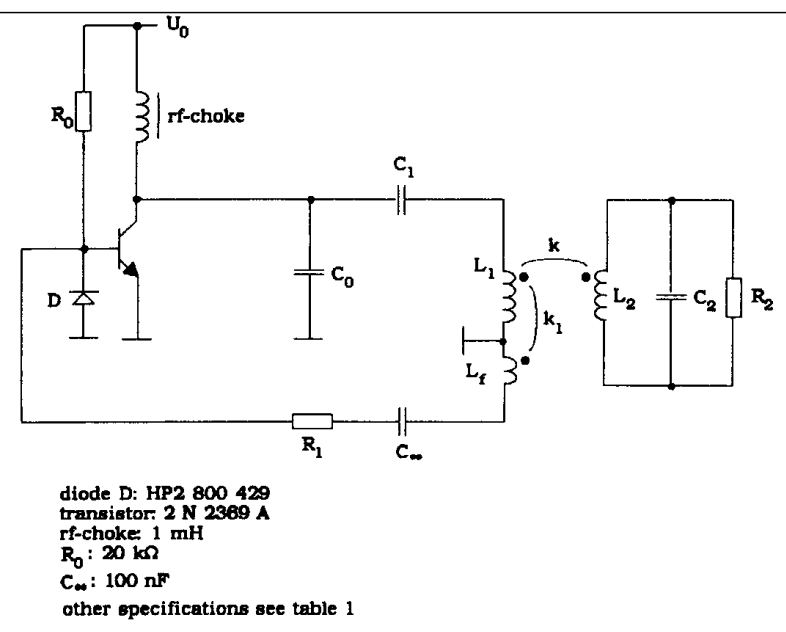

Fig. 1. Class E tuned power oscillator including an inductive link.

pled to a series tuned transmitter $\left(L_{1}, C_{1}\right)$. Oscillation is achieved by means of a feedback branch $\left(L_{f}, C_{\infty}, R_{1}\right)$. Diode $D$ and resistor $R_{0}$ provide proper bias voltage and ensure easy starting of oscillation.

The class $E$ concept efficiently permits conversion of dc-battery power to ac power at the switching frequency. A class E amplifier contains a single transistor, which is assumed to operate as a switch, i.e., collector-emitter voltage is essentially zero with nonzero collector current, and vice versa, collector current is about zero when collector-emitter voltage is applied. One basic condition for high efficiency operation is that the switch turn-on resistance of the transistor (i.e., collector-emitter saturation resistance $R_{\text {sat }}$ ) be comparatively small compared to the ohmic part $R$ of the input impedance $Z_{\text {in }}=R+j X$ of the class $E$ output circuit. In contrast to an ordinary class $E$ amplifier where resistance $R$ is equal to the load, $R$ in the present application is dependent on operating frequency $f_{0}$ and on the coupling factor $k$, i.e., $R=R\left(f_{0}, k\right)$. In the case of comparatively weak coupling of the coils (i.e., $0.1<k<0.3), R_{\text {sat }}$ is not negligible in comparison to $R\left(f_{0}, k\right)$ in the analysis.

\section{ANAlysis}

\section{A. Frequency Analysis}

To analyze the properties of the oscillator depicted in Fig. 1, the circuit is cut at the feedback branch and, in making investigations, it is assumed to be driven by an external frequency source (frequency analysis). An equivalent circuit is shown in Fig. 2.

This is based on the following assumptions:

- the RF choke allows only a constant (dc) input current $I_{0}$ and has no series resistance;

- the switching transistor can be described as an ideal switch in series with an ohmic resistor $R_{\text {sat }}$;

- the total shunt capacitance $C_{0}$ is independent of the collector-emitter voltage;

- resistor $R_{f}$ consists of resistor $R_{1}$ plus an additional

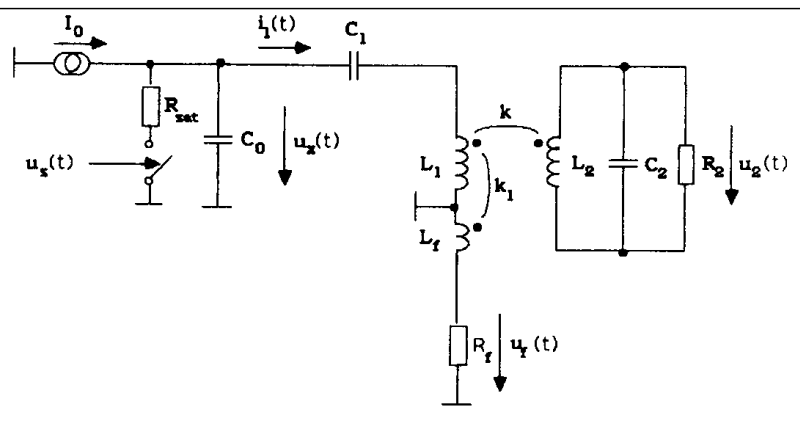

Fig. 2. Equivalent circuit for frequency analysis.

loss $\bar{R} . \bar{R}$ represents the ohmic resistance due to diode $D$, resistor $R_{0}$ and the base-emitter input resistor of the transistor switch. It is averaged over one time period.

Frequency analysis can be considered in two parts, i.e., into class $\mathrm{E}$ analysis and link analysis.

1) Class E Analysis: The analysis of the class E amplifier is performed using the above listed assumptions. The inductive link together with the feedback network is represented by the complex input impedance $Z_{\text {in }}$. In the following, the real and imaginary parts are designated as $R$ and $X$, i.e., $Z_{\text {in }}=R+j X$.

It is assumed that the series reactance $X$ applies to the fundamental frequency only and is infinite at harmonic frequencies. This allows the statement

$$
i_{1}(t)=i_{0} \sin (\omega t-\varphi)
$$

with amplitude $i_{0}$ and phase $\varphi$ to be determined. A time period $T$ is divided into two equal sections, (i.e., the duty ratio is $50 \%$ ), the transistor being switched off during the first section, and on during the second. Collector-emitter voltage $u_{x}(t)$ within the switch-off-state $(0<t<T / 2)$ then is

$$
\begin{aligned}
u_{x \mathrm{off}}(t) & =U_{x o}+\frac{1}{C_{0}} \int_{0}^{t} I_{0}-i_{1}(t) d t \\
& =U_{x o}+\frac{1}{\omega C_{0}}\left[I_{0} \omega t+i_{0} \cos (\omega t-\varphi)-\cos \varphi\right]
\end{aligned}
$$

with $U_{x o}$ as collector voltage at times $t=\cdots-2 t,-T$. $0, T, 2 T, \cdots$.

Determination of $u_{x}(t)$ in switch-on-state $(T / 2<t<$ $T$ ) may be achieved by means of Laplace transformation. The result is

$$
\begin{aligned}
u_{x \mathrm{on}}(t)= & I_{0} R_{\mathrm{sat}}-\frac{i_{0} R_{\mathrm{sat}}}{1+(\omega \tau)^{2}}[\sin (\omega t-\varphi) \\
& -\omega \tau \cos (\omega t-\varphi)] \\
& +\left[U_{x 1}-I_{0} R_{\mathrm{sat}}+\frac{i_{0} R_{\mathrm{sat}}}{1+(\omega \tau)^{2}}\right. \\
& \cdot(\sin \varphi+\omega \tau \cos \varphi)] \exp \left(\frac{\pi-\omega t}{\omega \tau}\right)
\end{aligned}
$$


with $U_{x 1}$ as collector voltage at times $t=\cdots 3 T / 2$, $-T / 2, T / 2,3 T / 3, \cdots$, and $\tau=R_{\text {sat }} \cdot C_{0}$. The third term of the sum describes the exponential discharging of capacitor $C_{0}$, when the switch is turned on.

Assuming $(\omega \tau)^{2} \ll 1$ yields
The corresponding coefficient $c_{1}^{\prime}$ of $u_{x}^{\prime}(t)$ is

$$
c_{1}^{\prime}=\frac{1}{T} \int_{0}^{T} u_{x}^{\prime}(t) \exp (-j \omega t) d t
$$

Setting $c_{1}=c_{1}^{\prime}$ yields, after comparison of real and imaginary parts, two equations containing $i_{0}$ and $\varphi$. Solving for $i_{0}$ and $\varphi$ gives

$$
\tan \varphi=\frac{\left(1+\frac{\pi}{2} \omega \tau\right) R+\frac{\pi}{2} X+\left(\frac{2}{\pi}-\frac{\pi}{4}+\frac{\omega \tau}{2}\right) \frac{1}{\omega C_{0}}}{-\left(1+\frac{\pi}{2} \omega \tau\right) X+\frac{\pi}{2} R+\left(\frac{1}{2}+\omega \tau\left(\frac{\pi}{2}+\frac{2}{\pi}\right)\right) \frac{1}{\omega C_{0}}}
$$

and

$$
\frac{i_{0}}{I_{0}}=\frac{1}{\left(\omega C_{0} X-\frac{1}{2}-\frac{2}{\pi} \omega \tau\right) \sin \varphi+\left(\omega C_{0} R+\frac{2}{\pi}+\frac{1}{2} \omega \tau\right) \cos \varphi}
$$

$$
\begin{aligned}
U_{x o}= & u_{x o n}(t=T) \approx I_{0} R_{\text {sat }}+i_{0} R_{\text {sat }}(\sin \varphi+\omega \tau \cos \varphi) \\
U_{x 1}= & u_{x \mathrm{off}}(t=T / 2)=U_{x o} \\
& +\left(I_{0} \pi-2 i_{0} \cos \varphi\right) /\left(\omega C_{0}\right) .
\end{aligned}
$$

Now collector-emitter voltage $u_{x}(t)$ can be completely determined for both halves of the period

$$
\begin{aligned}
u_{x \mathrm{fff}}(t)= & \frac{1}{\omega C_{0}}\left[I_{0} \omega(t+\tau)+i_{0}[\omega \tau \sin \varphi-\cos \varphi\right. \\
& +\cos (\omega t-\varphi)]] \\
u_{x \mathrm{on}}(t)= & \frac{1}{\omega C_{0}}\left[I_{0} \omega \tau-i_{0} \omega \tau \sin (\omega t-\varphi)\right. \\
& +i_{0}(\omega \tau)^{2} \cos (\omega t-\varphi) \\
& +\left[\pi I_{0}+2 i_{0} \omega \tau \sin \varphi-2 i_{0} \cos \varphi\right] \\
& \left.\cdot \exp \frac{\pi-\omega \tau}{\omega t}\right] .
\end{aligned}
$$

Amplitude $i_{0}$ and phase $\varphi$ are determined as follows: a sinusoidal current $i_{1}(t)$ would cause a sinusoidal voltage $u_{x}^{\prime}(t)$ over impedance $Z_{\text {in }}=R+j X$;

$$
u_{x}^{\prime}(t)=i_{0} R \sin (\omega t-\varphi)+i_{0} X \cos (\omega t-\varphi) .
$$

This fictitious voltage must be equal to the component of the Fourier series of the actual collector voltage $u_{x}(t)$ at the fundamental frequency. The complex Fourier coefficient $c_{1}$ is obtained with

$$
\begin{aligned}
c_{1}= & \frac{1}{T} \int_{0}^{T} u_{x}(t) \exp (-j \omega t) d t \\
= & \frac{1}{T}\left[\int_{0}^{T / 2} u_{x \mathrm{off}}(t) \exp (-j \omega t) d t\right. \\
& \left.+\int_{T / 2}^{T} u_{x \circ \mathrm{n}}(t) \exp (-j \omega t) d t\right]
\end{aligned}
$$

For the special case $R_{\text {sat }}=0$, these results agree with the corresponding results found in [9]. The supply voltage $U_{0}$ is equal to the dc component of $u_{x}(t)$. Using

$$
U_{0}=\frac{1}{T} \int_{0}^{T} u_{x}(t) d t
$$

and $i_{0} / I_{0}$ from above, dc-resistance $R_{\mathrm{dc}}=U_{0} / I_{0}$ can be estimated:

$$
\begin{aligned}
R_{\mathrm{dc}}= & \frac{1}{\omega C_{0}}\left[\left(\frac{\pi}{4}+\frac{3}{2} \omega \tau\right)+\frac{i_{0}}{I_{0}}\left(\left(\frac{\omega \tau}{2}+\frac{1}{\pi}\right)\right.\right. \\
& \left.\left.\cdot \sin \varphi-\frac{1}{2} \cos \varphi\right)\right] .
\end{aligned}
$$

Efficiency of this idealized class E scheme is determined by two effects, i.e., by the power consumption of resistor $R_{\text {sat }}$ in the on-state and by collector-emitter voltage $u_{x}(t)$ across capacitor $C_{0}$ at times $\cdots-3 T / 2,-T / 2, T / 2$, $3 T / 2 \cdots$ (i.e., $U_{x 1}$ ). The second loss mechanism can be minimized by proper circuit design.

2) Link Analysis: Link analysis is achieved with the help of Fig. 3. It depicts an equivalent circuit of the inductive link including the feedback branch.

To determine the overall two port describing the inductive link, two port $A$ (index $a$ ) containing all coupled coils first is evaluated. It is assumed that coil $L_{f}$ is just coupled to coil $L_{1}$ (coupling factor $k_{1}$ ), but not to coil $L_{2}$. Then impedance matrix $Z_{\text {aik }}$ becomes

$$
\begin{aligned}
Z_{\mathrm{aik}} & =\left[\begin{array}{ll}
Z_{a 11} & Z_{a 12} \\
Z_{a 21} & Z_{a 22}
\end{array}\right] \\
& =\left[\begin{array}{ll}
j \omega L_{1}+\omega^{2} k_{1}^{2} \frac{L_{1} L_{f}}{R_{f}+j \omega L_{f}} & j \omega k \sqrt{L_{1} L_{2}} \\
j \omega k \sqrt{L_{1} L_{2}} & j \omega L_{2}
\end{array}\right] .
\end{aligned}
$$




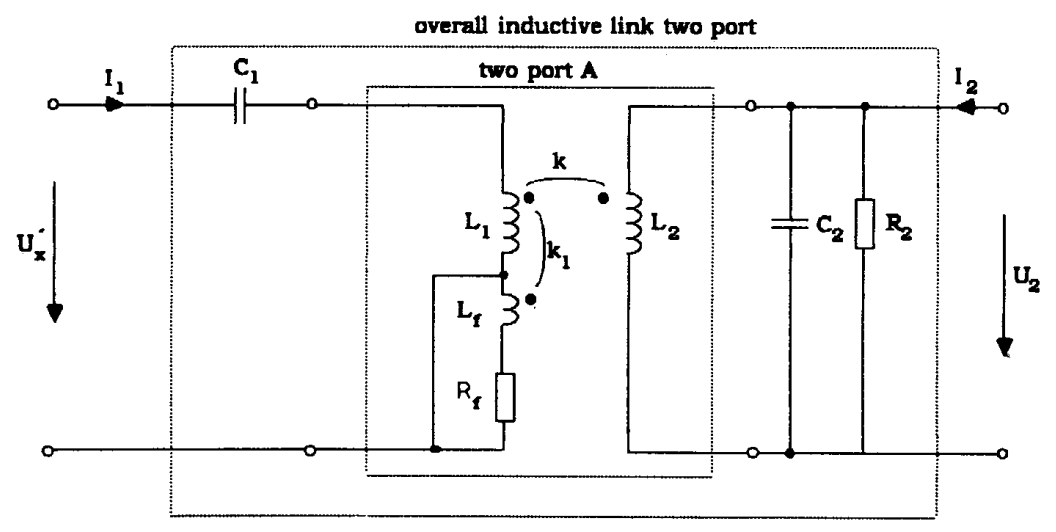

Fig. 3. Inductive link.

This matrix is transformed into the hybrid matrix $H_{\text {aik }}$ with relation

$$
H_{\mathrm{aik}}=\left[\begin{array}{ll}
H_{\mathrm{a} 11} & H_{\mathrm{a} 12} \\
H_{\mathrm{a} 21} & H_{\mathrm{a} 22}
\end{array}\right]=\frac{1}{Z_{\mathrm{a} 22}}\left[\begin{array}{cc}
\operatorname{det} Z_{\mathrm{aik}} & Z_{\mathrm{a} 12} \\
-Z_{\mathrm{a} 21} & 1
\end{array}\right] .
$$

The hybrid matrix $H_{i k}$ of the overall two port now can be simply obtained as

$$
\begin{aligned}
H_{i k} & =\left[\begin{array}{ll}
H_{11} & H_{12} \\
H_{21} & H_{22}
\end{array}\right] \\
& =\left[\begin{array}{ll}
H_{\mathrm{a} 11}+\frac{1}{j \omega C_{1}} & H_{\mathrm{a} 12} \\
H_{\mathrm{a} 21} & \left(H_{\mathrm{a} 12}+j \omega C_{2}+\frac{1}{R_{2}}\right)
\end{array}\right] .
\end{aligned}
$$

Using equations

$$
\begin{aligned}
U_{x}^{\prime} & =H_{11} I_{1}+H_{12} U_{2} \\
I_{2} & =H_{21} I_{1}+H_{22} U_{2}=0
\end{aligned}
$$

(complex pointers $I_{1}, I_{2}, U_{x}^{\prime}, U_{2}$ referring to Fig. 3) yields the input impedance $Z_{\text {in }}$ of the inductive link:

$$
Z_{\text {in }}=\operatorname{det}\left(H_{i k}\right) / H_{22} \text {. }
$$

Inserting $Z_{\text {in }}=R+j X$ into (9) and (10) now fully defines the class $\mathrm{E}$ amplifier and consequently the complete system. Output voltage $U_{2}$ and overall efficiency $\eta$ are calculated with matrix $H_{\mathrm{ik}}$ and (10) and (12):

$$
U_{2}=-\frac{H_{21}}{H_{22}} i_{0}=-\frac{H_{21}}{H_{22}} \frac{i_{0}}{I_{0}} \frac{U_{0}}{R_{\mathrm{dc}}}
$$

and

$$
\eta=\frac{\left|U_{2}\right|^{2}}{2 R_{2} U_{0} I_{0}}=\frac{1}{2 R_{2}}\left|\frac{H_{21}}{H_{22}}\right|^{2}\left(\frac{i_{0}}{I_{0}}\right)^{2} \frac{1}{R_{\mathrm{dc}}} .
$$

Function $\eta(f)$ is plotted in Fig. 4 for various coupling factors. The calculation is based on circuit specifications

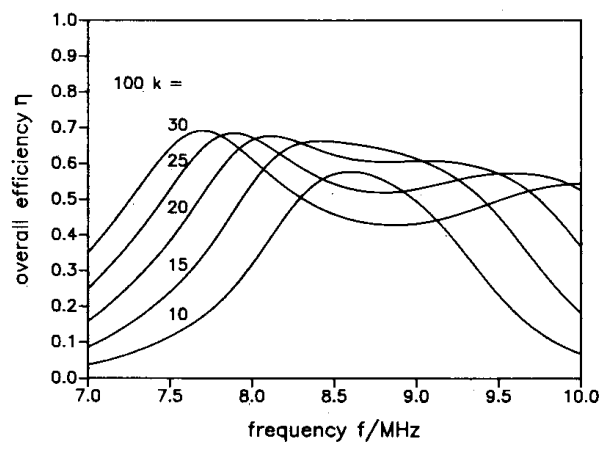

Fig. 4. Calculated overall efficiency $\eta(f)$ for various couplings.

TABLE I

CiRCUIT SPECIFICATIONS

\begin{tabular}{lcc}
\hline$U_{0}$ & 3.5 & $V$ \\
\hline$L_{1}$ & 2.35 & \\
$L_{2}$ & 3.4 & \\
$L_{f}$ & 0.42 & $\mathrm{H}$ \\
\hline$k_{\mathrm{l}}$ & 0.36 & \\
\hline$C_{0}$ & 213 & \\
$C_{1}$ & 217 & $\mathrm{pF}$ \\
$C_{2}$ & 101 & \\
\hline$R_{2}$ & 1500 & \\
$R_{f}$ & 200 & \\
$R_{\text {sat }}$ & 8 & \\
\hline
\end{tabular}

summarized in Table I. These values are also used for the following plots.

Fig. 4 shows that, for a given coupling, the absolute maximum efficiency is achieved in a frequency region between 7.5-8.5 MHz. Within this region, efficiency peaks move towards higher frequencies with decreasing coupling factor $k$.

\section{B. Oscillator Analysis}

Oscillation frequency $f_{o}$ of the class $\mathrm{E}$ oscillator is characterized by the fact that the phase shift between switch 


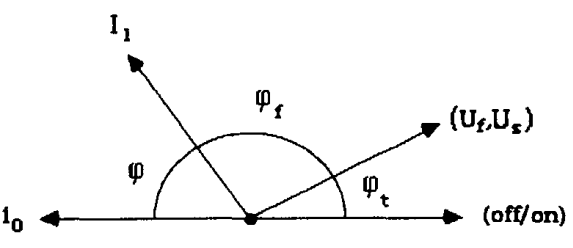

Fig. 5. Basic phase diagram

voltage $U_{s}$ and feedback voltage $U_{f}$ is zero (compare Fig. 2). Fig. 5 defines the phase relations of the circuit signals used to determine the oscillation frequency.

The pointer (off/on) indicates the actual switching phase of the transistor switch versus pointer $i_{0}$. The phase shift of $\pi$ between these pointers is due to the initial definition of $i_{1}(t)$ [compare (1)]. The phase shift $\varphi_{t}$ between pointers (off/on) and pointer $\left(U_{f}, U_{s}\right)$ represents a switching delay of the transistor versus base-emitter voltage $U_{f}$, i.e., $U_{s}$ (within the closed feedback configuration feedback voltage $U_{f}$ is identical to switching voltage $U_{s}$ ). This is due to dynamic effects within the transistor at high frequencies. Phase shift $\varphi_{f}$ can be estimated with the help of hybrid matrix $H_{i k}$. The result is

$$
\varphi_{f}=\pi-\operatorname{arctg} \frac{R_{f}}{\omega L_{f}} .
$$

Note, that both phase shifts $\varphi_{t}$ and $\varphi_{f}$ are independent of the coupling factor $k$.

Phase shift $\varphi(f)$ between pointers $i_{0}$ and $I_{1}$ is determined by (9). It is depicted in Fig. 6 as a function of operating frequency $f$ for various coupling factors $k$.

All curves $\varphi(f)$ intersect at point $S$. At this point, phase shift $\varphi(f)$ is independent of coupling factor $k$.

It can easily be proved that, given that two curves $\varphi(f)$ with different coupling intersect, then all curves do so: with the help of (15) and (17), (9) can always be reduced to a general form

$$
\operatorname{tg} \varphi=\frac{a(f)+k^{2} b(f)}{c(f)+k^{2} d(f)}
$$

with $a(f), b(f), c(f)$, and $d(f)$ as real polynomial functions of frequency $f$. Phase shift $\varphi(f)$ is independent of coupling $k$ if and only if

$$
\frac{a(f)}{b(f)}=\frac{c(f)}{d(f)}
$$

Each real solution of this algebraic equation defines an intersection point of all curves $\varphi(f)$ for arbitrary coupling factors $k$.

Oscillation frequency $f_{o}$ is determined by the following relation

$$
\pi-\varphi_{t}-\varphi_{f}=\varphi
$$

The left side of this equation is independent of coupling factor $k$ and comparatively weakly dependent on frequency $f$. In a first approximation it can be assumed to be

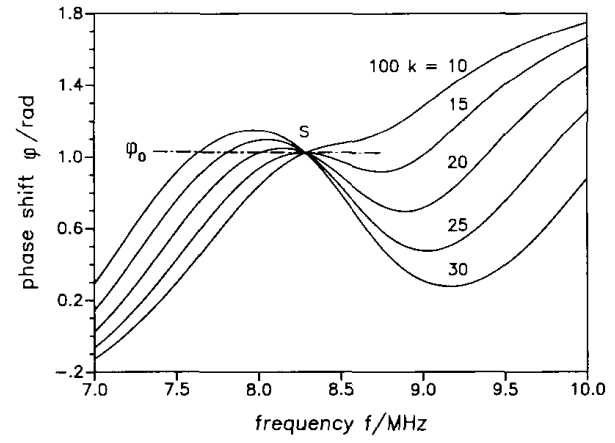

Fig. 6. Calculated phase shift $\varphi(f)$ for various couplings

constant within the operating frequency range, i.e.,

$$
\pi-\varphi_{t}-\varphi_{f} \approx \varphi_{0}=\text { constant }
$$

Operating points are defined by the intersection points of the straight line $\varphi_{0}$ and curves $\varphi(f)$. In this connection, intersection point $S$ plays an important role. Experimental and theoretic investigations show that the performance of the circuit in terms of overall efficiency and insensitivity of output voltage $U_{2}$ to coupling variations are optimized, if the straight line $\varphi_{0}$ defined above contains intersection point $S$.

If coupling is sufficiently high (compare Fig. 6, curves with $k=0.3,0.25,0.2,0.15$ ), there exist three intersection points of phase $\varphi(f)$ with the straight line $\varphi_{0}$. In this case point $S$ is not a stable operating point since

$$
\frac{\delta \varphi(f)}{\delta f}<0
$$

Two stable operating points, i.e., two possible oscillation frequencies for each solution, remain. If coupling drops below a certain limit, the only stable operating point is point $S$ (compare curve $\varphi(f)$ for $k=0.1$ of Fig. 6).

Which of the two stable operating points is preferred by the system is explained assuming that the coupling of the coils is being slowly increased. At low coupling the only stable operating point is $S$, as explained above. The coupling is now increased until the condition

$$
\frac{\delta \varphi(f)}{\delta f}=0
$$

is reached in point $S . S$ is still the only stable operation point. Any further increase of $k$ forces the system to decide between two intersection points left or right of $S$. $S$ itself is not stable, since (25) is valid. Theoretically neither right nor left is preferred; the probability is 0.5 for both cases. This decision can be avoided, if $S$ does not lie on straight line $\varphi_{0}$. E.g., if $S$ is somewhat below $\varphi_{0}$, the operating points of the system will be left of $S$ at increasing coupling. In every intersection point left of $S$ condition

$$
\frac{\delta \varphi(f)}{\delta f}>0
$$




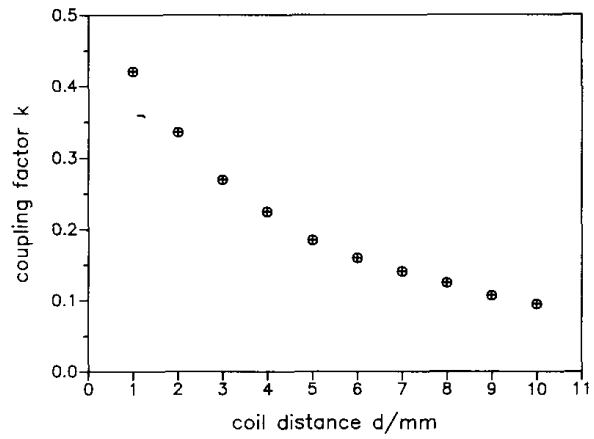

Fig. 7. Measured coupling factor $k$ as a function of spatial coil distance $d$.

is valid. If $S$ is above $\varphi_{0}$, the system will prefer operation points right of $S$. Thus in practical applications a left/right preference of the circuit operating points can be achieved by proper adjustment of $\varphi_{0}$.

The operating points situated to the left of point $S$ move towards higher frequencies, if coupling factor $k$ is decreased. A comparison with Fig. 4 shows that these operating points are tracking the absolute maximums of the overall efficiency. The behavior of the output voltage $U_{2}$ in terms of coupling variations is treated below. In the following, calculation and experimental results are compared. Fig. 7 depicts coupling factor $k$ measured as a function of spatial coil distance $d$.

Fig. 8 plots the following functions

- oscillation frequency $f_{0}$

- overall efficiency $\eta$ and

- output voltage $U_{2}$,

calculated and measured as a function of spatial coil distance $d$. Calculation is based on circuit specification of Table I and relationship $k(d)$ of Fig. 7. The feedback phase is determined by the location of point $S$. Calculation yields $\varphi_{0} / \mathrm{rad}=1.0278$.

Output voltage $U_{2}$ is almost independent of coupling up to a coil distance $d=8 \mathrm{~mm}$. Within this range overall efficiency varies between 60 and $70 \%$.

\section{Data Transmission}

In principle the system described can be used for inductive transmission of information. Practical schemes are

- amplitude modulation (AM) to convey an analog waveform, or

- amplitude shift keying (ASK) to transmit digital data. $\mathrm{AM}$ and ASK can be achieved to modulation of supply voltage $U_{0}$ of the transmitter circuit. A more detailed description of the AM and ASK performance of the circuit will be presented in a forthcoming paper.

\section{CONCLUSION}

The feature of the system described in the present paper may be summarized as follows:

1) A high overall efficiency is obtained, even if coil coupling is comparatively low. Thus heavy ferrite back-

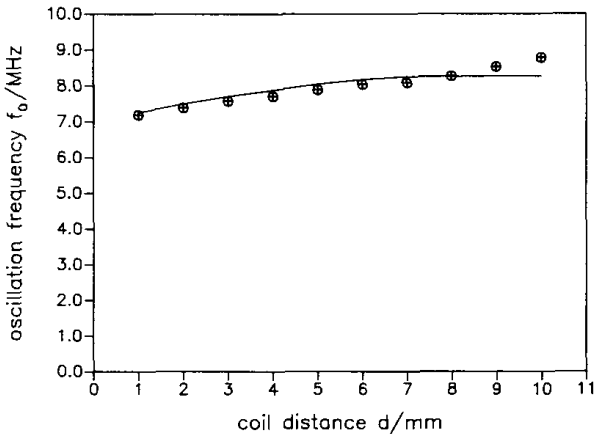

(a)

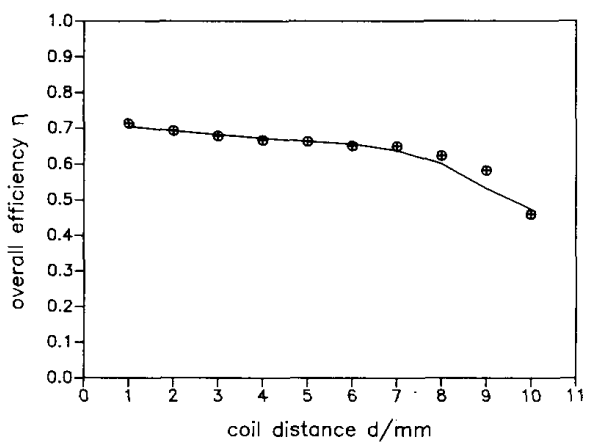

(b)

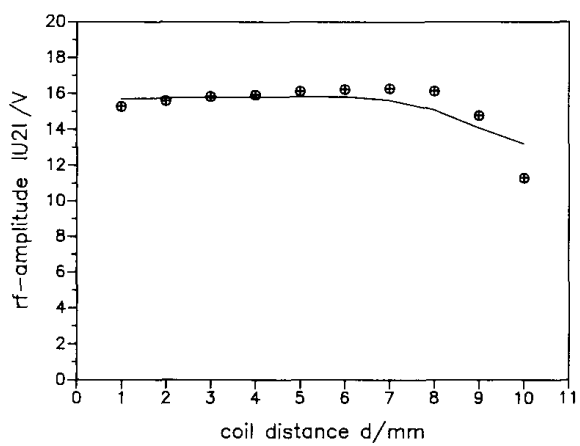

(c)

Fig. 8. (a) Oscillation frequency $f_{0}$. (b) Overall efficiency $\eta$. (c) Output voltage $U_{2} . \oplus$ indicates experimental results.

ings of the coils used in some applications to improve coil coupling [2] may be avoidable.

2) The RF voltage amplitude is extremely insensitive to coupling variations within a wide coupling range.

3) For realization of the transmission scheme a minimal number of circuit components is necessary. The only active element is a single transistor switch.

4) The operating frequency is not fixed, but influenced by the coil coupling.

5) Referring to point 4), a practical scheme for transmitting power and digital data, is e.g., amplitude shift keying. 


\section{REFERENCES}

[1] J. C. Shuder, H. E. Stephenson, and J. F. Townsend, "High-level electromagnetic energy transfer through a closed chest wall," in IRE Int Conv. Rec., part. 9, vol. 9, pp. 119-126, 1961

[2] D. C. Galbraith, M. Soma, and R. L. White, "A wide-band efficient inductive transdermal power and data link with coupling insensitive gain,"' IEEE Trans. Biomed. Eng., vol. BME-34, pp. 265-275, Apr. 1987.

[3] J. Steurer and E. Hochmair, "'Transcutane signal-und leistungsübertragung für implantate über hochfrequenz-bandfilter,'” Biomed. Tech., Band 31, Heft 4, 1986

[4] E. S. Hochmair, "System optimization for improved accuracy in transcutaneous signal and power transmission," IEEE Trans. Biomed. Eng., vol. BME-31, pp. 177-186, Feb. 1984

[5] W. H. Ko, S. P. Liang, and C. D. F. Fung, "Design of radio-frequency powered coils for implant instruments," Med. Biol. Eng. Comput., vol. 15, pp. 634-640.

[6] N. de N. Donaldson, and T. A. Perkins, "Analysis of resonant coupled coils in the design of radio-frequency transcutaneous links," Med. Biol. Eng. Comput., vol. 21, pp. 612-627, 1983.

[7] N. O. Sokal and A. D. Sokal, "Class E, a new class of high-efficiency tuned single-ended switching power amplifiers," IEEE $J$. Solid-State Circuits, vol. SC-10, no. 3, pp. 168-176, June 1975.

[8] N. O. Sokal, "Class E high-efficiency switching-mode tuned power amplifier with only one inductor and one capacitor in load networkapproximate analysis," IEEEJ. Solid-State Circuits, vol. SC-16, no. 4, Aug. 1981.

[9] F. H. Raab. "Idealized operation of the class $E$ tuned power amplifier," IEEE Trans. Circuits Syst., vol. CAS-24, pp. 725-735, Dec. 1977.

[10] "Effects of circuit variations on the class $\mathrm{E}$ tuned power amplifier," IEEE J. Solid-State Circuits, vol. SC-13, pp. 239-247, Apr. 1978.

[11] J. Ebert and M. Kazimierczuk, "Class E high efficiency tuned power oscillator," IEEE J. Solid-State Circuits, vol. SC-16, pp. 62-66, Apr. 1981 .

[12] M. Kazimierczuk, "Collector amplitude modulation of the class $\mathrm{E}$ tuned power amplifier," IEEE Trans. Circuits Syst., vol. CAS-31, vol. 6, pp. 543-549, June 1984.

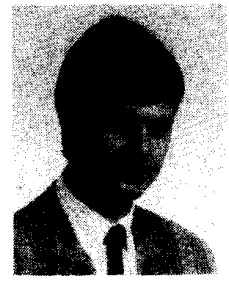

Clemens M. Zierhofer was born in Innsbruck Austria, in 1962 . He received the Dipl. Ing. and Dr.techn. degrees in electrical engineering from the Technical University of Vienna, Vienna, Aus tria, in 1985 and 1989 , respectively. His thesis was "An implant for combined analog and pulsatile electrostimulation of the acoustic nerve."

In 1986 he joined the Institute of Experimental Physics, University of Innsbruck, Austria. His research interests include cochlear implant design, signal processing and RF-power amplifiers.

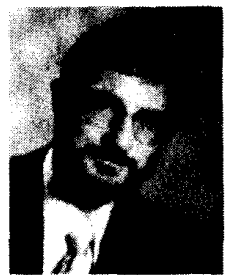

Erwin S. Hochmair was born in Vienna, Austria in 1940. He received the Dipl.-Ing, and Dr.techn. degrees in electrical engineering from the Technical University of Vienna, Vienna, Austria, in 1964 and 1967, respectively.

In 1965 he joined the Institute for Physical Electronics, Technical University of Vienna, where he teaches courses on linear integrated circuits and circuit design. From 1970 to 1972 he was with the NASA Marshall Space Flight Center, AL, as a Research Associate. During 1979 he was Visiting Associate Professor at Stanford University, Stanford, CA Since 1986 he has been a full professor at the Institute of Experimental Physics at the University of Innsbruck, Austria. His current research interests are circuit design, signal processing, and cochlear implant design. 